\title{
Survey Attention and Self-Reported Political Behavior
}

\author{
R. Michael Alvarez and Yimeng Li* \\ California Institute of Technology
}

December 2, 2021

\begin{abstract}
Survey research methodology is evolving rapidly, as new technologies provide new opportunities. One of the areas of innovation regards the development of online interview best practices, and the advancement of methods that allow researchers to measure the attention that subjects are devoting to the survey task. Reliable measurement of subject attention can yield important information about the quality of the survey response. In this paper, we take advantage of an innovative survey we conducted in 2018, in which we directly connect survey responses to administrative data, allowing us to directly assess the association between survey attention and response quality. We show that attentive survey subjects are more likely to provide accurate survey responses regarding a number of behaviors and attributes that we can validate with our administrative data. The best strategy to deal with inattentive respondents, however, depends on the correlation between respondent attention and the outcome of interest.
\end{abstract}

*We thank the John Randolph Haynes and Dora Haynes Foundation for supporting our research, and the Orange County Registrar of Voters for providing access to administrative data. We thank Jian Cao and Ines Levin for their collaborations with us on related projects. This paper was presented at the 2021 Midwest Political Science Association annual meeting; we thank panel participants for their comments and feedback about our research. 


\section{Introduction}

Survey research has been in the midst of vast changes in recent years, as new technologies provide new opportunities. New data sources can improve sampling and survey inference (Green and Gerber, 2006; Barber et al., 2014; Ghitza and Gelman, 2020), and researchers can contact and interview respondents using many different modes (Atkeson et al., 2014). New and sophisticated methods for weighting survey data are now available, helping researchers deal with the complexity of survey sampling and design (Caughey et al., 2020). And innovative new analytical methods allow researchers to use millions of survey responses, measured across many decades, to analyze opinion change (Caughey and Warshaw, 2018).

One of the primary issues is how these methodological changes might affect the quality of the survey response, which has been the subject of numerous recent studies (Meade and Craig, 2012; Ansolabehere and Schaffner, 2014; Atkeson et al., 2014; Maniaci and Rogge, 2014). Of particular interest has been whether the presence of an interviewer (say in a live telephone survey, or with an inperson interview) changes the incentives for survey respondents to pay close attention to survey questions and to provide accurate answers, which has been studied for decades by survey methodologists (Bradburn and Sudman, 1974). For example, survey methodologists have studied how interviewers may affect responses to certain types of survey questions, and whether respondents are more likely to provide more accurate information regarding controversial or sensitive questions when there is no interviewer present, especially for self-completion surveys (Tourangeau and Smith, 1996).

But with the increasing use of self-completion surveys, the absence of an 
interviewer may mean that respondents could move through a questionnaire quickly, and not pay close attention to the questions or the potential responses (Curran, 2016). Inattentive respondents in self-completion surveys may thus provide lower-quality data, as they might randomly answer questions, provide inaccurate responses, answer with a "don't know", or use other tactics to get through a survey quickly (Atkeson et al., 2014; Clifford and Jerit, 2015). These concerns have led researchers to study the use of "attention checks", "trap questions", "screeners", usually in the form of instructed response items or instructional manipulation checks (in this paper, we use the term attention checks).

There have been a number of recent studies that have examined the use of attention checks in surveys and opinion polls, documenting how attentive and inattentive survey respondents differ, studying different types of attention checks and methods for measuring attentiveness, and examining how to deal with inattentive respondents in survey data (Read et al., 2021; Berinsky et al., 2021; Kung et al., 2018; Hauser and Schwarz, 2015; Berinsky et al., 2014; Oppenheimer et al., 2009). There have also been studies that have looked at experimental subject attentiveness, in either convenience samples of students or from crowd-sourcing applications like MTurk (Ahler et al., 2021; Curran and Hauser, 2019; Curran, 2016; Thomas and Clifford, 2017). However, the lack of ground truth information prevents researchers from quantifying the performance of attention checks and comparing different approaches to dealing with inattentive respondents. In our paper, we attempt to fill this gap by examining responses to factual survey questions that we can validate with external administrative data.

Our results indicate that respondents failing attention checks are more likely to misreport various factual information. Many of these inattentive respondents 
nonetheless provide responses in line with the information in the administrative records. For turnout histories in recent elections, which correlate with respondent attention, dropping inattentive respondents leads to an unrepresentative subsample and, hence, estimates with larger biases and variances. By contrast, for modes of voting in recent elections, which are largely uncorrelated with attention check passages, dropping inattentive respondents yields estimates with smaller biases that often outweigh the cost of larger variances.

In the next section we discuss theory and past research, then the unique dataset and methodology that we use to test hypotheses about inattentive survey respondents. We next present results from our analysis, and conclude with a discussion of what our results imply for survey research.

\section{Theory and Past Research}

Inattentive respondents may diminish the quality of survey data. For example, one recent study found that inattentive respondents offer quick answers, are more like to give "don't know" answers, and are more likely to "straightline" (Alvarez et al., 2019). Theoretically, survey respondents often may engage in satisficing - which can occur when individuals encounter cognitively challenging tasks, and they do not expend the resources necessary to fully comprehend the question, to search their memory for the best answer, or otherwise provide only superficial attention to a survey question (Simon, 1956; Krosnick, 1991).

One solution to the problems caused by inattentive respondents is to use attention checks, also known as "screeners" or "trap questions". Past research has differentiated attention checks into two types. One type of attention check is an instructional manipulation check (IMC), where there is a deliberate change in 
the instructions in a survey question designed to capture whether the respondent is reading and cognitively processing the question's instructions (Oppenheimer et al., 2009; Berinsky et al., 2014; Anduiza and Galais, 2017). An example of an IMC is adding a clause to a survey question instructing the respondent to ignore the question and provide a specific answer. The other type of attention check is an instructed response item (IRI), where the responses to a survey question are altered in a way that should elicit whether the respondent is attentive to the question's response options (Alvarez et al., 2019). An example of an IRI is adding a row in a grid instructing respondents to select 'strongly disagree' for survey quality control.

However, due to the lack of ground truth information, previous studies have relied on various post-hoc measures to evaluate the performance of attention checks. ${ }^{1}$ These measures look at whether respondents passing and failing attention checks differ in producing canonical experimental results, empirical correlations between negatively correlated survey questions, straight-lining behavior, and response time. For the same reason, it has also been impossible to evaluate the performance of different statistical approaches to dealing with inattentive respondents. Our research takes advantage of a unique survey, which has questions that we can validate against administrative data, as well as measures of respondent attentiveness.

We motivate our research reported in this paper using the theory of satisficing (Simon, 1956; Krosnick, 1991). We hypothesize that inattentive survey respondents will provide less accurate answers to questions about their recent and past voting behavior, in particular whether they voted in the current and

\footnotetext{
${ }^{1}$ In a recent related study, Kuhn and Vivyan (2021) compared the accuracy of self-reported behavior using list experiments and direct questions, and comparing the responses to administrative data.
} 
past elections, and the method that they used to vote in the current and past elections (Hypothesis 1).

Because inattentive respondents provide less accurate answers, that generates error and noise in a survey dataset, so that when the data is used for modeling or testing hypotheses, including the answers from inattentive respondents will potentially generate bias in model estimates and incorrect inferences. Moreover, inattentive survey respondents who are engaging in satisficing will follow particular heuristics when they provide survey responses, like straightlining, midpoint responses, or socially desirable answers. Thus it is likely that inattentive respondents will generate systematic errors in a survey dataset, not just additional noise or variance. This leads to Hypothesis 2: inattentive respondents will provide answers that can introduce bias in model estimates.

The implications of our analysis are important for dealing with inattentive respondents in research using self-administered surveys. We find consistent support for Hypothesis 1, for self-reports of turnout for a number of recent elections, and for self-reports of the method that the voter used to return or cast their ballot. We also show support for Hypothesis 2, that there is evidence that inattentive respondents are generating systematic error in survey data (not random error). Thus respondent inattention will generate error and noise in survey self-reports of political behavior, and under the assumption that respondent attention is uncorrelated with the outcome of interest, we argue that dropping inattentives from the analysis will increase variance because of information loss, but will decrease bias in the estimates. However, under the assumption that respondent attention is correlated with the outcome of interest, if a researcher drops inattentive respondents that can produce bias by creating an unrepresentative 
sample (though the direction of the bias is not clear, and will depend on the type of heuristic that inattentive respondents use in answering the survey). Removing inattentive respondents in this situation will also increase variance.

Testing these hypotheses with ground-truth data is the gap in the literature that our research seeks to fill. In 2018 we fielded a unique survey that allowed us to connect survey responses to voter registration data. In our survey, we posed a series of questions, regarding current and past electoral participation as well as other demographic information, that we could validate with the administrative data. This provides us with a larger array of different types of ground-truth information, so that we can study the accuracy of inattentive and attentive survey respondents.

In the next section of our paper, discuss our survey design. Then we turn to various tests of our two hypotheses using data from our unique survey. We first examine how inattentive respondents answer questions about their voting participation and method of voting. We then examine our second hypothesis, and test for whether inattentive respondents are generating answers that introduce bias into models using their survey responses.

\section{Survey and Attention Checks}

Our study uses voter registration and voting history administrative data provided by the Orange County Registrar of Voters (OCROV). These datasets contain information about each registered voter in the county, including contact and demographic information, partisan registration, whether they turned out to vote in past elections, and if they did turn out in a past election whether they voted in person or by mail. Importantly, in California, voters can provide contact 
information (like a telephone number or email address) when they register to vote. Of the approximately 1.6 million voter registration records in the county in the 2018 general election, over 530,000 of those records were associated with an email address. We used all of the records with email addresses for our survey.

For our research on the 2018 general election in Orange County, we developed a self-completion online survey focused on the voter's election experiences. The online questionnaire included attention checks in the form of IMCs and IRIs, questions on voter registration, turnout in recent elections, and method of voting, in addition to questions on other subjects. We invited registered voters (via email) to participate in our survey between Thursday, November 8, 2018, and Tuesday, November 13, 2018. From 531,777 invites to all registered voters with email addresses, we received 6,952 complete responses. The survey took about 12 to 15 minutes to complete (the median duration was 13 minutes) and was provided in English. More details about the survey can be found in the paper's Supplementary Materials.

We then match back each survey response to the corresponding registered voter. In most cases $(6,816)$, the survey respondent can be linked back to a unique registered voter by the email address alone. ${ }^{2}$ In cases of ambiguity, we further match responses to voters according to age (or, in rare cases, a combination of age and gender) from self-reports and administrative records. By matching survey respondents directly to administrative data, we can validate self-reports of voter turnout and method of voting and add features (such as party registration) from the administrative data to our analysis. More details about the administrative data are discussed in the paper's Supplementary Materials.

\footnotetext{
${ }^{2}$ For each email address in the voter registration file, we generated a unique survey URL. This allows us to link the survey responses to records in the voter registration file when there is a unique record associated with the email address (6,816 out of 6,952 cases).
} 
Our 2018 survey contained both Instructional Manipulation Checks (IMC) and Instructed Response Items (IRI) for assessing survey attention. ${ }^{3}$ We designed the survey instrument so that respondents would receive one attention check approximately $25 \%$ of the way through the survey (attention check 1 ), the second when they had completed about $50 \%$ of their survey (attention check 2 ), and the final attention check at about $75 \%$ completed (attention check 3 ). In all cases, subjects who ignored the attention checks were allowed to continue to the next set of survey questions.

To avoid potential question order biases, we randomized the appearance of the attention check questions in our survey. For attention check 1, respondents were randomly assigned to receive either an IRI or IMC attention check. The IRI check asked subjects to answer "oppose" or "support" among five other questions about their opinions regarding election reform; the IMC attention check asked subjects to ignore a question on news media websites and to select two specific news organizations as their answers.

Attention check 2 followed a series of questions about the subject's voting experiences. Those who had been asked to answer the IRI for attention check 1 were then asked the IMC for attention check 2. Similarly, subjects who answered the IMC for attention check 1 were asked the IRI attention check. Again, we designed this to avoid any question order or location effects with respect to the use of the IMC or IRI format as attention checks.

Finally, the third attention check was located about $75 \%$ of the way through the survey questionnaire. Here the subjects were again randomized, with half receiving an additional IRI, while the others received an additional IMC. We

\footnotetext{
${ }^{3}$ We used an IMC studied in Berinsky et al. (2014) and an IRI studied in Alvarez et al. (2019) as our main attention checks.
} 
Table 1: IMCs Screen Respondents More Aggressively Than IRIs

\begin{tabular}{lrcc}
\hline & Fail & Pass & Skip \\
\hline IRI - Attention check 1 or 2 & $15 \%$ & $82 \%$ & $4 \%$ \\
IRI - Attention check 1 & $16 \%$ & $81 \%$ & $4 \%$ \\
IRI - Attention check 2 & $13 \%$ & $83 \%$ & $4 \%$ \\
Additional IRI & $9 \%$ & $88 \%$ & $4 \%$ \\
IMC - Attention check 1 or 2 & $45 \%$ & $52 \%$ & $3 \%$ \\
IMC - Attention check 1 & $49 \%$ & $47 \%$ & $4 \%$ \\
IMC - Attention check 2 & $40 \%$ & $57 \%$ & $3 \%$ \\
Additional IMC & $28 \%$ & $70 \%$ & $2 \%$ \\
\hline
\end{tabular}

included this attention check (an additional IRI or an additional IMC) for the purposes of robustness checks; we present our results from the main IRI and IMC attention checks in the text. ${ }^{4}$

Questions on turnout and mode of voting in the 2018 General Election appear right before attention check 1 , while questions on turnout and mode of voting in previous elections appear right after attention check 2 . We provide the complete wording of the attention checks and these questions in the paper's Supplementary Materials.

The overall passage rates of our IRI and IMC attention checks are presented in Table 1. The first four rows of the Table provide statistics on failure rates for the IRI questions, while the last four rows show similar statistics on the failure rates for the IMC questions. Consistent with previous studies on self-administered

\footnotetext{
${ }^{4}$ In the third attention check, the IRI asked subjects to answer either "disagree strongly" or "agree strongly" after a battery of questions about political polarization. The final attention check's IMC involved a question about social media; subjects were asked to ignore the question and select two specific answers to show they are paying attention to the survey questions.
} 
online surveys (Berinsky et al., 2014; Alvarez et al., 2019), a significant fraction of respondents failed these attention checks. As we expected, IMCs screen respondents more aggressively than IRIs, with $45 \%$ of respondents failing the IMC at either attention check 1 or 2 and 15\% of respondents failing the IRI at either attention check 1 or 2.

Also, it is important to note two other results in Table 1. First, regardless of whether a respondent receives the IRI or the IMC question at attention check 2 (after they have had one of the attention checks earlier in the survey), we see that the passage rates for both are slightly higher later in the survey. Similarly, for the third attention check, again, passage rates are higher as well for both types of attention check questions. This indicates that as subjects proceed through the survey, the presence of successive attention checks may increase the cognitive focus of survey respondents. ${ }^{5}$

Furthermore, the passage of our IRI and IMC attention checks is correlated with important demographics, as shown in Table SM1 in the Supplementary Materials. Educational attainment is positively correlated with the passage of each type of attention check, consistent with previous studies on self-administered online surveys (Berinsky et al., 2014; Alvarez et al., 2019). Non-white respondents are less likely to pass IRI and IMC attention checks than white respondents, again consistent with previous research. Male and female voters appear equally likely to pass the attention checks. Finally, contrary to previous studies, we find that age is negatively correlated with the passage of each type of attention check.

\footnotetext{
${ }^{5}$ These results require further and future study, but are beyond the scope of the research we report in this paper.
} 


\section{Results}

Before turning to our main results, we first look at three pieces of basic respondent information that we are able to validate using the administrative records (presented in Table SM2 in the Supplementary Materials): year of birth, city of residence, and voter registration before or after January 1, 2017, For each of the three pieces of basic respondent information and each of the two types of attention checks, respondents failing either the IRI or IMC attention check are more likely to misreport the information than those passing either attention check.

We begin by testing Hypothesis 1, that inattentive survey respondents will provide less accurate answers to questions about their recent and past voting behavior. Here we focus on whether they voted in the current and past elections, and the method that they used to vote in the current and past elections. Our first step is to compare the accuracy of self-reported turnout between respondents passing and failing each type of attention check, as shown in Figure 1. The analysis reported in Figure 1 takes advantage of the fact that we can ask respondents for self-reports about whether they participated in a number of previous elections, and that we can then check the veracity of their self-reports against our administrative data.

It's important to note that we consistently see over-reporting of turnout in our data. But for the purposes of testing Hypothesis 1 , the immediate observation from Figure 1 is that respondents failing either the IRI or the IMC attention check are significantly more likely to misreport turnout than those passing either attention check. While the magnitude of the difference is modest for the reporting of turnout in the 2018 general election (a few days before the survey), 
Figure 1: Inattentive Respondents Are More Likely to Misreport Turnout
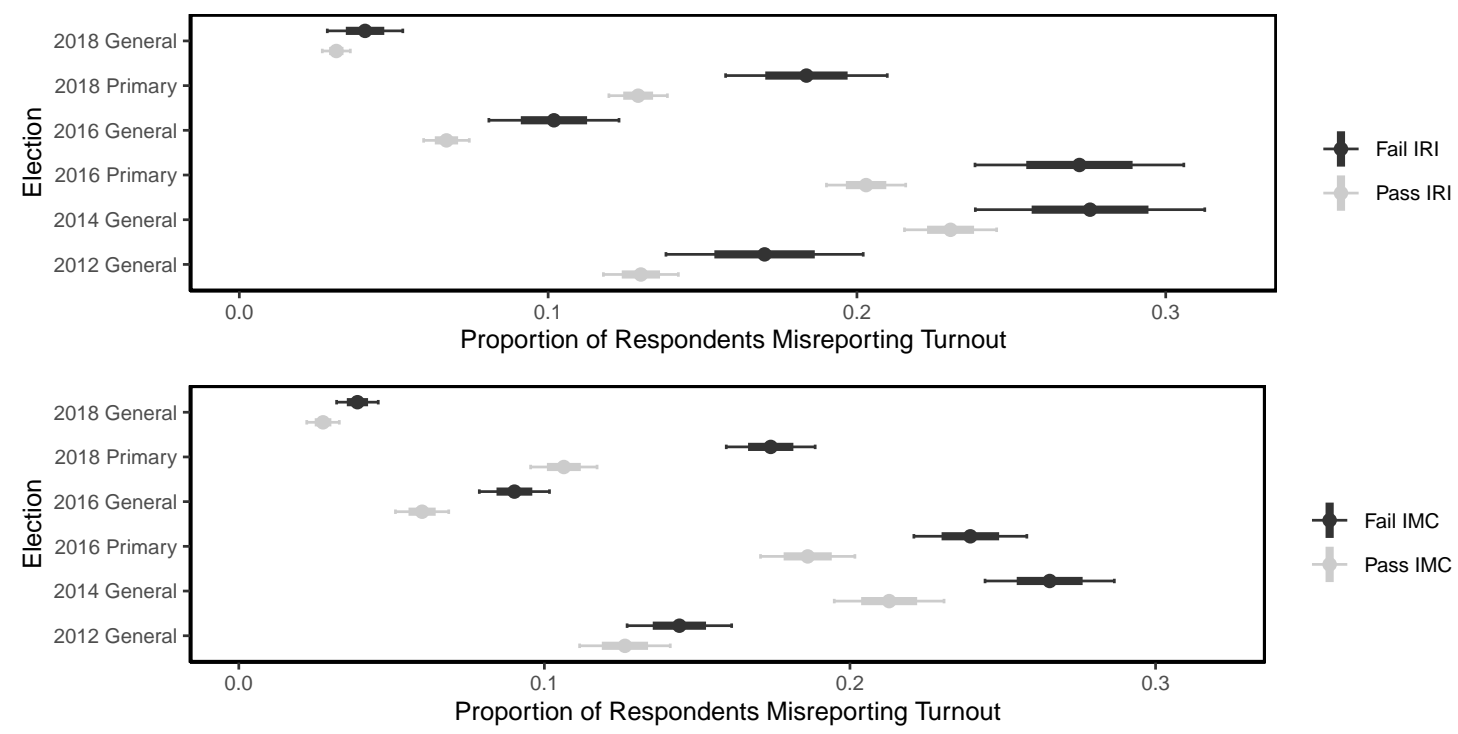

Note: This figure displays the proportion of respondents misreporting turnout in six recent elections among those who failed the IRI (top panel, black), passed the IRI (top panel, grey), failed the IMC (bottom panel, black), or passed the IMC (bottom panel, grey). In each case, dots display the point estimates, thin intervals display $95 \%$ confidence intervals, and thick intervals display $90 \%$ confidence intervals.

the difference is generally sizable for many past elections. This is not surprising, because trying to remember participation in past elections requires greater cognitive effort (especially primary elections, which are less salient compared to general elections), as subjects need to search their memory more extensively in order to provide a correct report. For example, $18.4 \%$ and $17.4 \%$ of respondents failing the IRI and IMC, respectively, misreported turnout in the 2018 Primary Election, compared to $12.9 \%$ and $10.6 \%$ who passed these attention checks.

These results indicate that including inattentive respondents as measured by these attention checks will lead to bias in the estimation of turnout. Also evident from Figure 1, however, is that many respondents who failed the attention checks but provided accurate self-reports of turnout nonetheless. This 
observation implies that dropping inattentive respondents as measured by these attention checks will increase the variance in the estimation of turnout. Finally, notice that respondents passing IMC provided the most accurate account of turnout, followed by respondents passing IRI, and then all respondents. This observation is consistent with the pattern that IMC screens out respondents more aggressively than IRI.

Our administrative data also contains information about the method that each voter used to cast their ballot in past elections, and in our survey, we asked each respondent to recall their method of voting. Our second test of Hypothesis 1 examines the difference in terms of accurately reporting the method of voting between respondents passing and failing each type of attention check, with results presented in Figure 2. Again, here we see consistent misreporting in our self-reported survey data. While the differences seen in this figure are less pronounced than the difference shown in Figure 1 for the accuracy of reported turnout, respondents failing IRIs are significantly more likely to misreport mode of voting than those passing IRIs, in all of the elections except the 2014 General Election. The same is true for IMC, but only significant for more recent elections.

One interesting pattern seen in Figure 2 is that generally the extent of misreporting is greater for elections further in the past, than for the most recent election. This pattern suggests that recall of the method that a voter used to obtain and return their ballot might be cognitively demanding for voters, in particular some of them who are not paying close attention as they complete the questionnaire.

The magnitude of the accuracy difference between self-reports of mode of voting by respondents failing and passing the attention checks averages around 
Figure 2: Inattentive Respondents Are More Likely to Misreport Mode of Voting


Note: This figure displays the proportion of respondents misreporting mode of voting in six recent elections among those who failed the IRI (top panel, black), passed the IRI (top panel, grey), failed the IMC (bottom panel, black), or passed the IMC (bottom panel, grey). In each case, dots display the point estimates, thin intervals display $95 \%$ confidence intervals, and thick intervals display $90 \%$ confidence intervals.

$1.8 \%$ and $1.0 \%$ for IRI and IMC, respectively, for the past two election cycles. This result indicates that including inattentive respondents as measured by these attention checks will lead to bias in the estimation of mode of voting, modest but statistically significant in most cases. As is the case with turnout, we find that many voters reported mode of voting accurately regardless of attentiveness. This pattern suggests that dropping inattentive respondents will lead to less precise estimates of mode of voting. Ultimately, the bias-variance trade-off will govern the best strategy to deal with inattentive respondents, which we shall explore in greater detail later.

So far, we have used validated survey responses to establish that inattentive respondents provided less accurate reports of turnout and method of voting, 
Figure 3: Respondent Attention Is Positively Correlated with Validated Turnout


Note: This figure displays the proportion of respondents confirmed to have participated in six recent elections among those who failed the IRI (top panel, black), passed the IRI (top panel, grey), failed the IMC (bottom panel, black), or passed the IMC (bottom panel, grey). In each case, dots display the point estimates, thin intervals display $95 \%$ confidence intervals, and thick intervals display $90 \%$ confidence intervals.

both for contemporary and past elections. Our findings are consistent with previous research that found inattentive and attentive respondents provide different self-reports of key political behavior and attitudes (for example, Alvarez et al. 2019). While these results highlight the bias in the estimation of political behavior if respondent inattention is not accounted for, dealing with inattentive respondents is complicated by the fact that respondent attention may be correlated with political behavior and attitudes of interest.

Our second hypothesis regards this issue, that inattentive respondents will provide answers that can introduce bias in model estimates. We test our second hypothesis and its corollaries in a number of ways in Figures 3- 6. First, Figure 3 presents the correlation between respondent attention and turnout by com- 
paring validated turnout between respondents passing and failing each type of attention check. Clearly, respondents failing the attention check are significantly less likely to turn out to vote than those passing the attention check, for both IRI and IMC. The magnitude of the difference ranges from 1.6\% (2018 General) to $8.0 \%$ (2018 Primary) for IRI and $1.0 \%$ (2012 General) to 4.5\% (2016 Primary). Our result is the first to document the positive correlation between respondent attention and political participation using validated turnout. ${ }^{6}$ The implication is that dropping inattentive respondents, a common way to address respondent inattention, can introduce a source of bias by creating a sample unrepresentative of the population in terms of political participation. Again, consistent with the fact that the IMC screens out respondents more aggressively than the IRI, respondents passing the IMC had the highest level of turnout, followed by the IRI, and then all respondents.

We next examine the correlation between respondent attention and mode of voting by comparing validated mode of voting between respondents passing and failing each type of attention check in Figure 4. In contrast with turnout, respondents failing the attention check are not different from those passing the check in terms of their actual choice of voting method. This result indicates that dropping inattentive respondents will not introduce bias that comes from an unrepresentative sample in terms of mode of voting. As we discuss in detail below, the presence or absence of correlation between respondent attention and political behavior or attitudes of interest dictates the magnitude of this important source of bias and ultimately factors heavily into the consideration of strategies to deal with respondent inattention.

\footnotetext{
${ }^{6}$ Alvarez et al. (2019) found positive correlations between respondent attention and selfreported political participation.
} 
Figure 4: Respondent Attention Is Not Correlated with Validated Mode of Voting


Note: This figure displays the proportion of respondents confirmed to have voted by mail in six recent elections among those who failed the IRI (top panel, black), passed the IRI (top panel, grey), failed the IMC (bottom panel, black), or passed the IMC (bottom panel, grey). In each case, dots display the point estimates, thin intervals display $95 \%$ confidence intervals, and thick intervals display $90 \%$ confidence intervals.

With these results in hand, we can now work toward developing the best strategy to deal with inattentive respondents in different scenarios. To illustrate the considerations going into such decisions, we consider the simplest possible strategy, dropping inattentive respondents identified by the attention checks. We first compare the performance of turnout estimates based on all respondents, respondents passing the IRI, and respondents passing the IMC, in terms of bias, standard error, and root mean squared error, as shown in Figure 5. While respondents failing the attention checks are significantly more likely to misreport turnout (Figure 1), the reduction in bias from dropping these inattentive respondents is countervailed by the increase in bias from the unrepresentativeness of attentive respondents in terms of turnout (Figure 3). As 
Figure 5: Dropping Inattentive Respondents Does Not Reduce Bias in Turnout Estimates
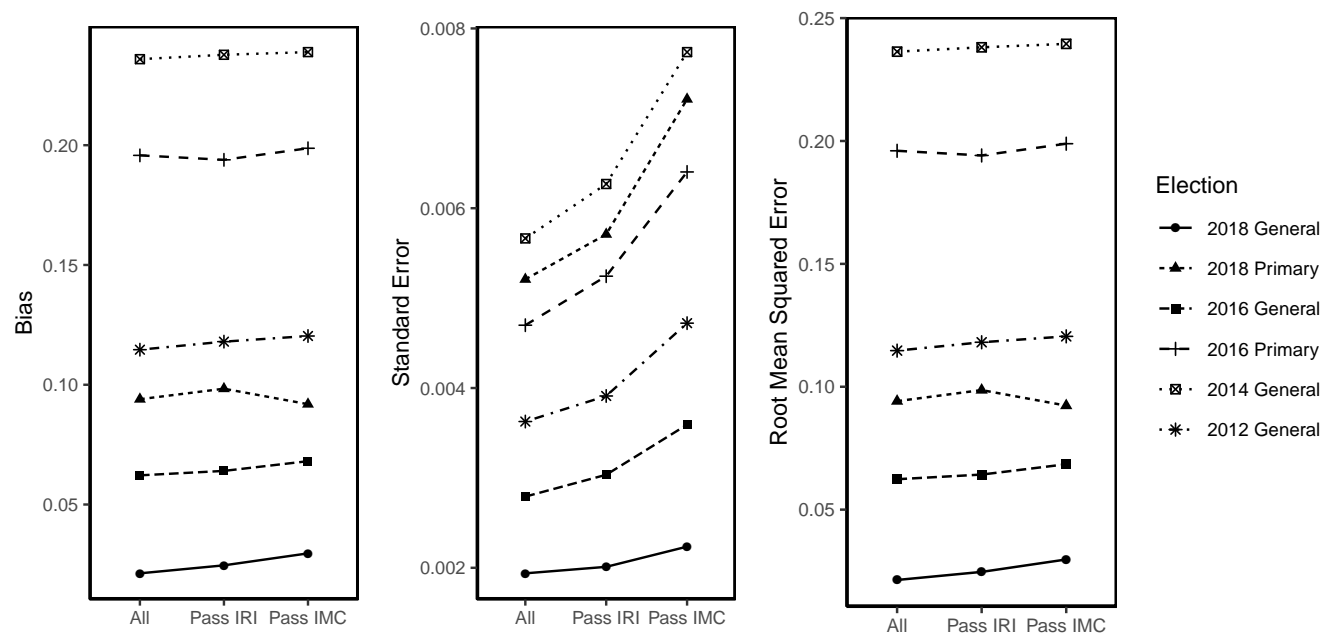

Note: This figure displays the bias (left panel), standard error (middle panel), and root mean squared error (right panel) of turnout estimates based on all respondents, respondents passing the IRI, and respondents passing the IMC, for six recent elections.

a result, dropping inattentive respondents does not reduce bias in turnout estimates (left panel). Respondents failing the attention checks, moreover, often provide correct self-reports of turnout nonetheless, leading to the pattern that keeping all respondents yields turnout estimates of the smallest standard errors (middle panel). Given the patterns present in terms of bias and variance, it's unsurprising that we find that the turnout estimates based on all respondents have the smallest root mean squared errors in most cases (right panel).

We next look at the difference in the performance of estimates of proportions of by-mail voters based on all respondents as well as respondents passing each type of attention check, with results presented in Figure 6. In contrast to the results for turnout, since respondent attention as measured by attention checks is uncorrelated with their mode of voting, dropping inattentive respondents 
Figure 6: Dropping Inattentive Respondents Reduces Bias in Voting-by-Mail Estimates
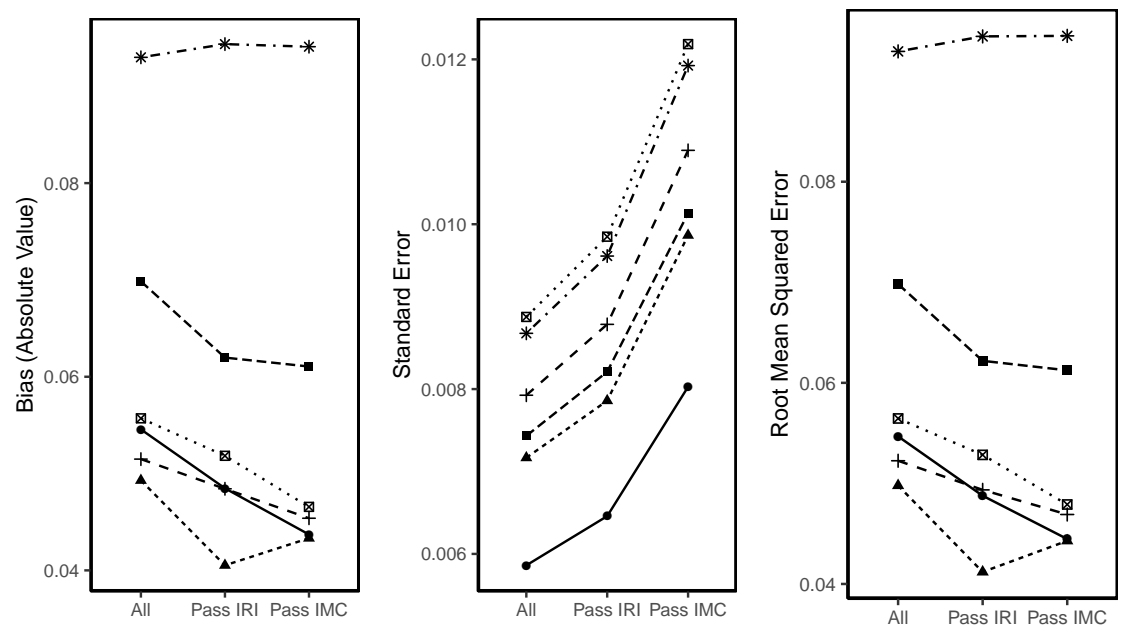

Election

- 2018 General

- 2018 Primary

-F. 2016 General

+ 2016 Primary

- $\bullet 2014$ General

*. 2012 General

Note: This figure displays the bias (left panel), standard error (middle panel), and root mean squared error (right panel) of estimates of proportions of by-mail voters based on all respondents, respondents passing the IRI, and respondents passing the IMC, for six recent elections.

does not create an unrepresentative sub-sample that would lead to an increase in bias. Dropping respondents failing attention checks, therefore, leads to a reduction of bias (left panel, with the sole exception of 2012 General) as these respondents are more likely to misreport their mode of voting. While such procedures still increase imprecision of the estimates compared to keeping all respondents (middle panel), its effect is dwarfed by the reduction of bias. Dropping inattentive respondents, as a result, yield smaller root mean squared errors in estimates of proportions of by-mail voters (right panel). Notice that dropping inattentive respondents reduce bias due to the higher likelihood of misreporting from these respondents and increase variance due to a reduced sample size in both turnout estimates and the estimates of proportions of by- 
mail voters. The difference in the best strategies for these two quantities of interest comes from the correlation, or the lack thereof, between respondent attention and outcomes of interest, or equivalently, the representativeness of inattentive respondents for the entire sample in terms of the variable of interest.

\section{Discussion and Conclusion}

The implications of our analysis are important for dealing with inattentive respondents in research using self-administered surveys. We find consistent support for Hypothesis 1, that attentive survey subjects are more likely to provide accurate survey responses, for self-reports of turnout for a number of recent elections, and for self-reports of the method that the voter used to return or cast their ballot. We also show support for Hypothesis 2, that there is evidence that inattentive respondents are generating systematic (not random) error in survey data.

We obtained these results using a large sample from a diverse population, and our findings are consistent with the results in studies that have focused on attentiveness in small samples (for example, student or MTurk studies). Our paper, meanwhile, advances survey research in this area by validating survey responses with external administrative data at the individual level, and is the first paper to do so to the best of our knowledge. Thus we argue that our results have general and important implications for survey research, in particular for large-scale surveys and polls. Our focus on specific factual questions (voter registration, turnout, mode of voting) is driven by our ability to validate the responses to these questions in our administrative data. We leave to future research to explore other factual questions that can be validated using other 
forms of external ground-truth information.

However, the question that we are left with, since we know that there are inattentive subjects in survey studies, is what should a researcher do? One analytic strategy is to simply drop the inattentive subjects from any analysis of survey data. This approach often is implemented during the course of survey research, when subjects who do not pass a particular attention check are not allowed to continue with the remainder of the survey. As we have argued, based on our research, this approach is problematic. If under the assumption that respondent attention is uncorrelated with the outcome of interest, dropping inattentives will increase variance because of information loss, but will decrease bias in the estimates. But if respondent attention is correlated with the outcome of interest, dropping inattentive respondents can produce an unrepresentative sample, and thus bias estimates. While the direction of the bias is not clear, and will depend on the type of heuristic that inattentive respondents use in answering the survey, bias will be an issue in these situations. As it is often not possible to know which assumption is valid, applied researchers should proceed with caution regarding how they deal with inattentive respondents.

Our advice is that survey researchers should avoid dropping inattentive respondents from their analyses, as either increasing variance or producing bias should be avoided. Rather, researchers should use methods that allow them to incorporate the heterogeneity in survey response generated by inattentive respondents - which, if done correctly, can avoid increasing variance and producing bias. Minimally, researchers should test results of survey research for robustness with respect to survey attentiveness, and if there is evidence of potential bias, then include measures of survey attention (either from direct questions 
or proxies) in models of survey responses. More research is necessary to help develop best practices for measuring attention and for modeling the heterogeneity induced by differences in attention.

Our research design also allows us to compare different types of attention checks. Between instructed response items and instructional manipulation checks, two commonly used types of attention checks, we find that instructional manipulation checks screen respondents more aggressively than instructed response items. As a result, while using instructional manipulation checks as screeners further reduces biases compared to instructed response items in many (but not all) cases, it comes at the cost of larger variances for our estimates. For applied researchers, we suggest considering multiple factors. First, while the variance consideration is secondary for a large survey like ours (over 6,900 respondents), it may be a primary consideration for surveys of small or moderate sizes. Secondly, many survey instruments already contain grid/list questions, making instructed response items much less costly than instructional manipulation checks, which require additional standalone questions just for quality control purposes. Lastly, since multiple attention checks are often recommended for online surveys longer than a few minutes, different types of attention checks can be employed to ensure the robustness of results to various levels of respondent attention screening. 


\section{References}

Ahler, D. J., C. E. Roush, and G. Sood (2021). The micro-task market for lemons: data quality on amazon's mechanical turk. Political Science Research and Methods, 1-20.

Alvarez, R. M., L. R. Atkeson, I. Levin, and Y. Li (2019). Paying attention to inattentive survey respondents. Political Analysis 27(2), 145-162.

Anduiza, E. and C. Galais (2017). Answering without reading: Imcs and strong satisficing in online surveys. International Journal of Public Opinion Research 29(3), 497-519.

Ansolabehere, S. and B. F. Schaffner (2014). Does survey mode still matter? findings from a 2010 multi-mode comparison. Political Analysis 22(3), 285-303.

Atkeson, L. R., A. N. Adams, and R. M. Alvarez (2014). Nonresponse and mode effects in self- and interviewer-administered surveys. Political Analysis 22(3), 304-320.

Barber, M., C. Mann, J. Monson, and K. Patterson (2014). Online polls and registrationbased sampling: A new method for pre-election polling. Political Analysis 22, 321-335.

Berinsky, A. J., M. F. Margolis, and M. W. Sances (2014). Separating the shirkers from the workers? making sure respondents pay attention on self-administered surveys. American Journal of Political Science 58(3), 739-753.

Berinsky, A. J., M. F. Margolis, M. W. Sances, and C. Warshaw (2021). Using screeners to measure respondent attention on self-administered surveys: Which items and how many? Political Science Research and Methods 9(2), 430-437.

Bradburn, N. M. and S. Sudman (1974). Response effects in surveys: A review and synthesis. Chicago: Aldine Publishing Company.

Caughey, D., A. J. Berinskey, S. Chatfield, E. Hartman, E. Schickler, and J. S. Sekhon (2020). Target Estimation and Adjustment Weighting for Survey Nonresponse and Sampling 
Bias. Elements in Quantitative and Computational Methods for the Social Sciences. Cambridge University Press.

Caughey, D. and C. Warshaw (2018). Policy preferences and policy change: Dynamic responsiveness in the american states, 1936-2014. American Political Science Review 112(2), 249-266.

Clifford, S. and J. Jerit (2015). Do attempts to improve respondent attention increase social desirability bias? Public Opinion Quarterly 79(3), 790-802.

Curran, P. G. (2016). Methods for the detection of carelessly invalid responses in survey data. Journal of Experimental Social Psychology 66, 4-19.

Curran, P. G. and K. A. Hauser (2019). I'm paid biweekly, just not by leprechauns: Evaluating valid-but-incorrect response rates to attention check items. Journal of Research in Personality 82, 103849.

Ghitza, Y. and A. Gelman (2020). Voter registration databases and mrp: Toward the use of large-scale databases in public opinion research. Political Analysis 28(4), 507-531.

Green, D. P. and A. S. Gerber (2006). Can registration-based sampling improve the accuracy of midterm election forecasts? Public Opinion Quarterly 70(2), 197-223.

Hauser, D. J. and N. Schwarz (2015). It'sa trap! instructional manipulation checks prompt systematic thinking on "tricky” tasks. Sage Open 5(2), 2158244015584617.

Kim, S.-y. S., S. Schneider, and R. M. Alvarez (2020). Evaluating the quality of changes in voter registration databases. American Politics Research 48(6), 670-676.

Krosnick, J. A. (1991). Response strategies for coping with the cognitive demands of attitude measures in surveys. Applied cognitive psychology 5(3), 213-236. 
Kuhn, P. M. and N. Vivyan (2021). The misreporting trade-off between list experiments and direct questions in practice: Partition validation evidence from two countries. Political Analysis, 1-22.

Kung, F. Y., N. Kwok, and D. J. Brown (2018). Are attention check questions a threat to scale validity? Applied Psychology 67(2), 264-283.

Maniaci, M. R. and R. D. Rogge (2014). Caring about carelessness: Participant inattention and its effects on research. Journal of Research in Personality 48, 61-83.

Meade, A. W. and S. B. Craig (2012). Identifying careless responses in survey data. Psychological methods 17(3), 437.

Oppenheimer, D. M., T. Meyvis, and N. Davidenko (2009). Instructional manipulation checks: Detecting satisficing to increase statistical power. Journal of experimental social psychology 45(4), 867-872.

Read, B., L. Wolters, and A. J. Berinsky (2021). Racing the clock: Using response time as a proxy for attentiveness on self-administered surveys. Political Analysis.

Simon, H. A. (1956). Rational choice and the structure of the environment. Psychological review 63(2), 129.

Thomas, K. A. and S. Clifford (2017). Validity and mechanical turk: An assessment of exclusion methods and interactive experiments. Computers in Human Behavior 77, $184-197$.

Tourangeau, R. and T. W. Smith (1996). Asking sensitive questions: The impact of data collection mode, question format, and question context. Public opinion quarterly 60(2), 275-304. 


\section{Supplementary Materials}

\section{Details about Administrative Data}

\section{Administrative Data}

Given the importance of administrative data for the work we report in this paper, here we provide additional details for interested readers. Administrative data, like voter registration records, have long been used by political science researchers. Some use voter registration records to provide contact information for field experiments (e.g., Gerber and Green 2000), as sampling frames to improve the accuracy of electoral polling (e.g. Green and Gerber 2006), or to study the misreporting of voter turnout in surveys (e.g., Ansolabehere and Hersch 2017). These studies all assume that the administrative records they use are accurate.

In the United States, states are now generally required to have a statewide voter registration database due to the Help America Vote Act (HAVA, 2002). These statewide voter registration datasets are used for many purposes, other than academic research. Campaigns and political parties use them for voter mobilization and persuasion (Hersch 2015). State, county, and municipal election officials use these data for voter information activities, to send vote-by-mail ballots, to allocate resources for in-person voting, and to authenticate eligible voters during an election. Clearly election officials have important incentives to keep these data as accurate as possible.

The procedures that states can use to maintain the accuracy of their voter registration datasets is regulated by the National Voter Registration Act (NVRA, 1993). Each state then issues it's own mechanisms for compliance with NVRA, and California's rules are provided by the Secretary of State (https://www.sos.ca. 
gov/elections/voter-registration/nvra/laws-standards/nvra-manual). In general, the NVRA regulations provide guidance about how potentially ineligible voters can be moved to an inactive status or removed from the file, usually based on changes like moving outside of the jurisdiction or death. In our paper we use data from Orange County, and their registration list maintenance procedures are provided online (https://www.ocvote.com/registration/ maintaining-an-accurate-voter-list).

As has been noted in recent research using these administrative data from Orange County (Kim et al., 2020), in a jurisdiction of this size, there will be record changes, new records, and removed records. However, this recent research has shown that these changes in the administrative data are relatively small in relation to the overall file, and there is no research that we are aware of that would indicate that file maintenance or inaccuracies in the administrative data more generally should have any effect on our estimate of the quantities we examine in our paper. In particular, the components of the voter registration data that are most subject to change and possible error are the fields with voter identifying information - their name, address, and other voter-provided information. The information from the administrative files we used in our paper, each registered voter's participation in current and past elections, comes from the county's election management system and we have every reason to believe that these components of the administrative record are accurate.

By focusing our research on a large and diverse county, we argue that our results should generalize. Orange County, California, is a very large and diverse election jurisdiction located in Southern California. With about 3 million residents, in the 2018 general election Orange County had just over 2 million citizens 
eligible to vote, with nearly 1.6 million registered voters. ${ }^{7}$. It is one of the largest election jurisdictions in the United States, closely divided in partisan registration: in 2018, the county had 541,665 registered Republicans, 523,624 registered Democrats, and 429,675 registered No Party Preference voters.

\section{Details about the Survey}

Among 6,952 respondents who completed our survey, we are able to determine basic demographic information for 6,912 respondents. Figure 7 shows the demographic compositions and the distributions of other individual characteristics for our survey respondents and the population of Orange County registered voters before the November 2018 general election.

Registered voters of different ages and genders are well represented in our survey sample. There are slightly more (2.7\%) respondents between 30 and 44 years old, fewer (4.4\%) respondents from age group 45-64, and fewer (2.0\%) female respondents, compared to the population of registered voters. Our survey sample exhibits more imbalances in race/ethnicity and party registration. More white voters and fewer Hispanic or Latino and Asian American voters participated in the survey (by $8.7 \%, 6.1 \%$ and $6.0 \%$ respectively). Meanwhile, while $34.7 \%$ of voters registered with the Republican party in Orange County, only $24.0 \%$ of those who completed our survey are Republican voters. On the other hand, $44.6 \%$ of respondents are Democratic voters, compared to $33.5 \%$ in the population. The disparities in terms of race/ethnicity and party registration in our survey sample are expected given our knowledge about survey participation in general and consistent with other surveys with voluntary participation. Fi-

\footnotetext{
${ }^{7}$ https://elections.cdn.sos.ca.gov/sov/2018-general/sov/02-county-voter-reg-stats-by-county. pdf
} 
Figure 7: Respondent Composition of the Survey

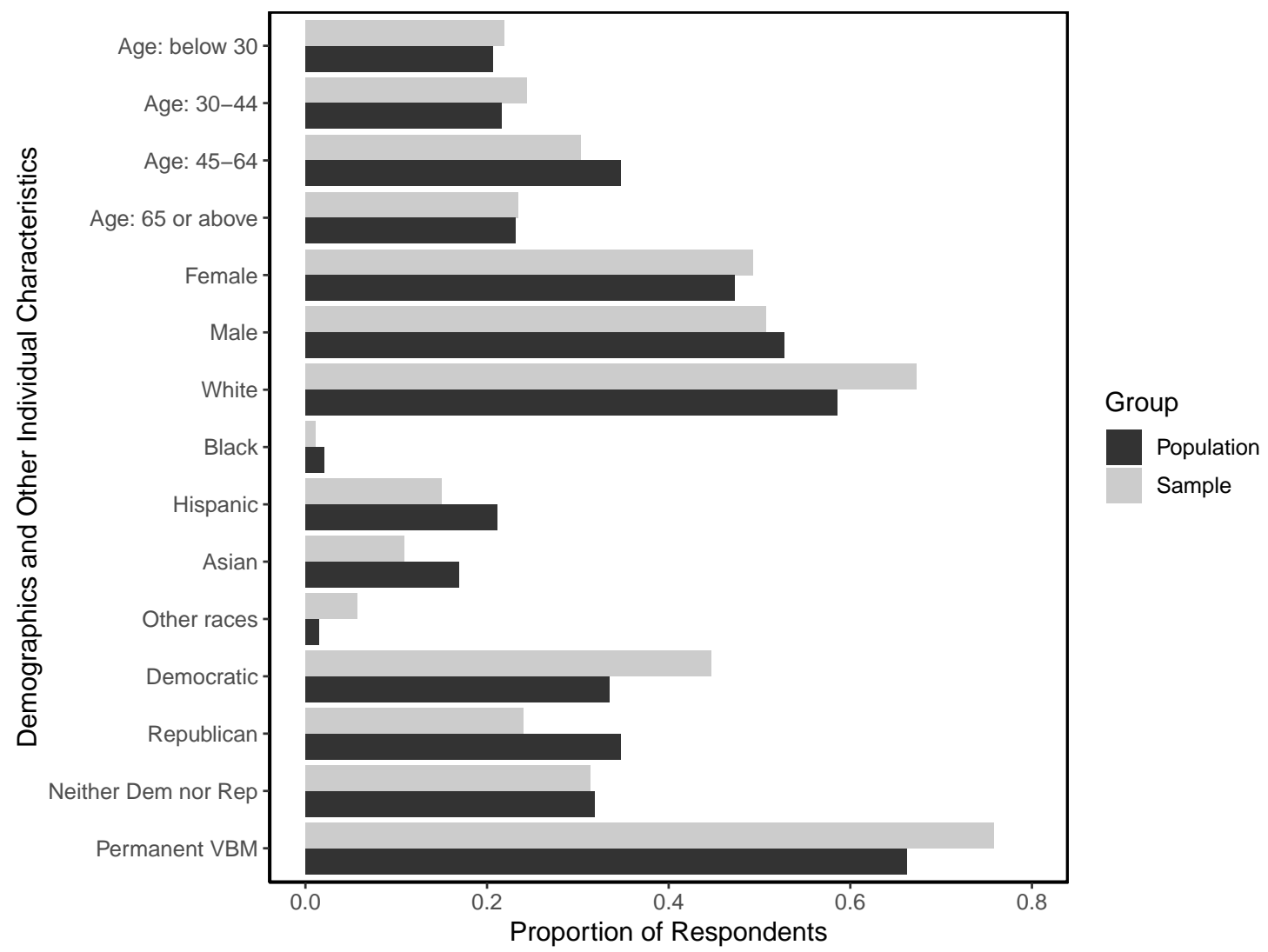

nally, $66.2 \%$ of registered voters in Orange County are permanent vote-by-mail voters, whereas the percentage is $75.8 \%$ in our sample; the distribution of cities of residence for our sample tracks the population well.

Of course, we could use calibration weighting to adjust our sample to population parameters (Caughey et al. 2020). We decided here to use unweighted data, as we did not want to make population estimates specific to Orange County. That said, our unweighted estimates should generalize to other similar large-scale surveys and polls. Weighting to Orange County population parameters would not change the general results we see in our analysis, and as Orange County is 
a large and diverse population, our results should apply to surveys and polls of other large and diverse populations.

\section{Attention Check Questions}

Main IRI: Do you support or oppose any of the following proposals for new ways of voting or conducting elections?

[For each of the following statements, respondents can select Support strongly, Support somewhat, Neither support nor oppose, Oppose somewhat, Oppose strongly]

- Allow absentee voting over the Internet

- Run all elections by mail

- Allow people to register on Election Day at the polls

- Require all people to show government issued photo ID when they vote

- Require electronic voting machines to print a paper backup of the ballot

- For half of the respondents: For survey quality control, select Oppose strongly

- For half of the respondents: For survey quality control, select Support strongly

Note: To minimize order effects, response options are presented in ascending order for half of the respondents, and in descending order for the other half of the respondents. 
Main IMC: When a big news story breaks people often go online to get up-to-theminute details on what is going on. People differ in which websites they trust to get this information. For survey quality control, please ignore the question and select $\mathrm{ABC}$ News and Reuters as your two answers.

When there is a big news story, which websites you would visit first?

- New York Times website

- The Drudge Report

- The Associated Press (AP) website

- Huffington Post

- Google News

- NBC News website

- Washington Post website

- ABC News website

- National Public Radio (NPR) website

- CNN.com

- CBS News website

- USA Today website

- FoxNews.com

- Reuters website

- New York Post Online 
- MSNBC.com

- Yahoo! News

- None of these websites

Note: The position of prompt For survey quality control, please ignore the question and select $A B C$ News and Reuters as your two answers is randomized between the middle of the question and the end of the question. 


\section{Questions on Turnout and Mode of Voting}

Note: Turnout Question 1 (2018 General Turnout), Turnout Question 2a, and Mode of Voting Question 1 (2018 General Mode of Voting) appear right before the first attention check, while Turnout Question 2b, Turnout Question 3 (Turnout in Previous Elections) and Mode of Voting Question 2 (Mode of Voting in Previous Elections) appear right after the second attention check.

Turnout Question 1: Which of the following statements best describes you?

- I did not vote in the election this November

- I thought about voting this time, but didn't

- I usually vote, but didn't this time

- I tried to vote, but was not allowed to when I tried

- I tried to vote, but it ended up being too much trouble

- I definitely voted in the November General Election

[If the respondent chose one of the last three response options in Turnout Question 1 , they receive the following two questions.]

Turnout Question 2a Was this your first time voting, or have you voted in elections before? [Randomize first two options.]

- I am a first time voter

- I have voted in elections before

- I don't know

Mode of Voting Question 1 How did you vote this election? 
- Voted in person on Election Day (at a polling place or precinct)

- Voted in person before Election Day

- Voted by mail or absentee ballot by mail

- I don't know

[If the respondent chose one of the first three response options in Turnout Question 1, they receive the following question.]

Turnout Question 2b Have you voted in elections before? [Randomize first two options.]

- Yes

- No

- I don't know

[If the respondent chose I have voted in elections before in Turnout Question 2a or Yes in Turnout Question 2b, they receive the following questions.]

Turnout Question 3 Earlier you mentioned that you have voted in elections before. Did you vote in the following general elections? [For each of the following statements, respondents can select Yes, No, or I don't remember.]

- November 2016 Presidential General Election

- November 2014 Midterm General Election

- November 2012 Presidential General Election

... in the following primary elections?

- June 2018 Statewide Primary Election 
- June 2016 Presidential Primary Election

Mode of Voting Question 2 How did you vote in these general elections you voted in? [For each of the following statements, respondents can select Voted in person, Voted by mail or absentee ballot by mail, or I don't know. Only those elections that a respondent answers Yes to in Turnout Question 3 are included.]

- November 2016 Presidential General Election

- November 2014 Midterm General Election

- November 2012 Presidential General Election

How did you vote in the primary elections you voted in?

- June 2018 Statewide Primary Election

- June 2016 Presidential Primary Election 
Table SM1: Demographics and Passage of Attention Checks (Logistic Regression)

\begin{tabular}{lcccc}
\hline & \multicolumn{2}{c}{ IRI } & \multicolumn{2}{c}{ IMC } \\
& Avg. ME & Std. Error & Avg. ME & Std. Error \\
\hline Below 30 & & & & \\
$30-44$ & -0.03 & 0.01 & -0.03 & 0.02 \\
$45-64$ & -0.07 & 0.01 & -0.11 & 0.02 \\
65 or above & -0.12 & 0.01 & -0.16 & 0.02 \\
Female & & & & \\
Male & 0.00 & 0.01 & -0.02 & 0.01 \\
Rather not say & -0.04 & 0.05 & -0.08 & 0.06 \\
Self-describe & 0.02 & 0.05 & -0.06 & 0.07 \\
HS or less & & & & \\
Some college & 0.06 & 0.03 & 0.13 & 0.03 \\
2-year college & 0.03 & 0.03 & 0.16 & 0.04 \\
4-year college & 0.08 & 0.03 & 0.19 & 0.03 \\
Postgraduate & 0.11 & 0.03 & 0.24 & 0.03 \\
White & & & & \\
Hispanic & -0.12 & 0.02 & -0.15 & 0.02 \\
Asian & -0.11 & 0.02 & -0.09 & 0.02 \\
Black & -0.19 & 0.05 & -0.17 & 0.06 \\
Other races & -0.08 & 0.02 & -0.23 & 0.03 \\
\hline
\end{tabular}


Table SM2: Accuracy of Self-Reported Birth Year, City of Residence, and Voter Registration

\begin{tabular}{lrrrr}
\hline & Incorrect & Correct & Skip & N \\
\hline Birth Year: & & & & \\
Fail IRI & $6.3 \%$ & $92.9 \%$ & $0.8 \%$ & 999 \\
Pass IRI & $3.4 \%$ & $96.0 \%$ & $0.6 \%$ & 5648 \\
Fail IMC & $4.8 \%$ & $94.8 \%$ & $0.4 \%$ & 3072 \\
Pass IMC & $3.2 \%$ & $96.2 \%$ & $0.6 \%$ & 3618 \\
\hline City of Residence: & & & & \\
Fail IRI & $8.4 \%$ & $90.9 \%$ & $0.6 \%$ & 971 \\
Pass IRI & $6.8 \%$ & $92.8 \%$ & $0.4 \%$ & 5474 \\
Fail IMC & $7.9 \%$ & $91.7 \%$ & $0.4 \%$ & 2994 \\
Pass IMC & $6.8 \%$ & $92.9 \%$ & $0.3 \%$ & 3496 \\
\hline Voter Registration: & & & & \\
Fail IRI & $8.5 \%$ & $88.0 \%$ & $3.4 \%$ & 986 \\
Pass IRI & $6.9 \%$ & $90.9 \%$ & $2.2 \%$ & 5608 \\
Fail IMC & $8.3 \%$ & $88.7 \%$ & $3.0 \%$ & 3037 \\
Pass IMC & $6.3 \%$ & $92.0 \%$ & $1.8 \%$ & 3595 \\
\hline
\end{tabular}


Table SM3: Inattentive Respondents Are More Likely to Misreport Turnout

\begin{tabular}{lccccc}
\hline & All & Fail IRI & Pass IRI & Fail IMC & Pass IMC \\
\hline 2018 General & 0.033 & 0.041 & 0.031 & 0.039 & 0.028 \\
& $(0.002)$ & $(0.006)$ & $(0.002)$ & $(0.003)$ & $(0.003)$ \\
2018 Primary & 0.137 & 0.184 & 0.129 & 0.174 & 0.106 \\
& $(0.004)$ & $(0.013)$ & $(0.005)$ & $(0.007)$ & $(0.006)$ \\
2016 General & 0.073 & 0.102 & 0.067 & 0.09 & 0.06 \\
& $(0.004)$ & $(0.011)$ & $(0.004)$ & $(0.006)$ & $(0.004)$ \\
2016 Primary & 0.212 & 0.272 & 0.203 & 0.239 & 0.186 \\
& $(0.006)$ & $(0.017)$ & $(0.007)$ & $(0.009)$ & $(0.008)$ \\
2014 General & 0.236 & 0.275 & 0.23 & 0.265 & 0.213 \\
& $(0.007)$ & $(0.019)$ & $(0.008)$ & $(0.011)$ & $(0.009)$ \\
2012 General & 0.135 & 0.17 & 0.13 & 0.144 & 0.126 \\
& $(0.006)$ & $(0.016)$ & $(0.006)$ & $(0.009)$ & $(0.008)$ \\
\hline
\end{tabular}


Table SM4: Inattentive Respondents Are More Likely to Misreport Mode of Voting

\begin{tabular}{lccccc}
\hline & All & Fail IRI & Pass IRI & Fail IMC & Pass IMC \\
\hline 2018 General & 0.055 & 0.072 & 0.053 & 0.061 & 0.053 \\
& $(0.003)$ & $(0.008)$ & $(0.003)$ & $(0.004)$ & $(0.004)$ \\
2018 Primary & 0.091 & 0.105 & 0.089 & 0.095 & 0.085 \\
& $(0.005)$ & $(0.014)$ & $(0.005)$ & $(0.007)$ & $(0.006)$ \\
2016 General & 0.119 & 0.135 & 0.118 & 0.129 & 0.111 \\
& $(0.005)$ & $(0.014)$ & $(0.005)$ & $(0.008)$ & $(0.007)$ \\
2016 Primary & 0.116 & 0.133 & 0.115 & 0.118 & 0.114 \\
& $(0.006)$ & $(0.017)$ & $(0.007)$ & $(0.009)$ & $(0.008)$ \\
2014 General & 0.124 & 0.123 & 0.126 & 0.126 & 0.124 \\
& $(0.007)$ & $(0.018)$ & $(0.008)$ & $(0.011)$ & $(0.009)$ \\
2012 General & 0.161 & 0.199 & 0.157 & 0.165 & 0.155 \\
& $(0.007)$ & $(0.021)$ & $(0.008)$ & $(0.011)$ & $(0.01)$ \\
\hline
\end{tabular}


Table SM5: Respondent Attention Is Positively Correlated with Validated Turnout

\begin{tabular}{lccccc}
\hline & All & Fail IRI & Pass IRI & Fail IMC & Pass IMC \\
\hline 2018 General & 0.952 & 0.939 & 0.955 & 0.944 & 0.958 \\
& $(0.003)$ & $(0.008)$ & $(0.003)$ & $(0.004)$ & $(0.003)$ \\
2018 Primary & 0.707 & 0.638 & 0.718 & 0.683 & 0.727 \\
& $(0.006)$ & $(0.016)$ & $(0.006)$ & $(0.009)$ & $(0.008)$ \\
2016 General & 0.894 & 0.865 & 0.9 & 0.878 & 0.907 \\
& $(0.004)$ & $(0.012)$ & $(0.005)$ & $(0.007)$ & $(0.005)$ \\
2016 Primary & 0.688 & 0.639 & 0.698 & 0.665 & 0.71 \\
& $(0.007)$ & $(0.018)$ & $(0.007)$ & $(0.01)$ & $(0.009)$ \\
2014 General & 0.622 & 0.561 & 0.635 & 0.601 & 0.64 \\
& $(0.007)$ & $(0.02)$ & $(0.008)$ & $(0.011)$ & $(0.01)$ \\
2012 General & 0.835 & 0.789 & 0.84 & 0.83 & 0.84 \\
& $(0.006)$ & $(0.017)$ & $(0.007)$ & $(0.009)$ & $(0.008)$ \\
\hline
\end{tabular}


Table SM6: Respondent Attention Is Not Correlated with Validated Mode of Voting

\begin{tabular}{lccccc}
\hline & All & Fail IRI & Pass IRI & Fail IMC & Pass IMC \\
\hline 2018 General & 0.681 & 0.666 & 0.685 & 0.678 & 0.689 \\
& $(0.006)$ & $(0.015)$ & $(0.006)$ & $(0.009)$ & $(0.008)$ \\
2018 Primary & 0.661 & 0.638 & 0.664 & 0.666 & 0.657 \\
& $(0.007)$ & $(0.021)$ & $(0.008)$ & $(0.011)$ & $(0.01)$ \\
2016 General & 0.62 & 0.625 & 0.622 & 0.618 & 0.626 \\
& $(0.007)$ & $(0.019)$ & $(0.008)$ & $(0.011)$ & $(0.01)$ \\
2016 Primary & 0.613 & 0.619 & 0.611 & 0.611 & 0.61 \\
& $(0.009)$ & $(0.023)$ & $(0.009)$ & $(0.013)$ & $(0.012)$ \\
2014 General & 0.603 & 0.593 & 0.601 & 0.613 & 0.598 \\
& $(0.01)$ & $(0.027)$ & $(0.011)$ & $(0.015)$ & $(0.013)$ \\
2012 General & 0.556 & 0.559 & 0.555 & 0.547 & 0.558 \\
& $(0.009)$ & $(0.024)$ & $(0.01)$ & $(0.014)$ & $(0.012)$ \\
\hline
\end{tabular}


Table SM7: Dropping Inattentive Respondents Does Not Reduce Bias in Turnout Estimates

\begin{tabular}{lccc}
\hline & All & Pass IRI & Pass IMC \\
\hline Bias: & & & \\
2018 General & 0.021 & 0.025 & 0.03 \\
2018 Primary & 0.094 & 0.098 & 0.092 \\
2016 General & 0.062 & 0.064 & 0.068 \\
2016 Primary & 0.196 & 0.194 & 0.199 \\
2014 General & 0.236 & 0.238 & 0.239 \\
2012 General & 0.115 & 0.118 & 0.12 \\
\hline Std. Error: & & & \\
2018 General & 0.002 & 0.002 & 0.002 \\
2018 Primary & 0.005 & 0.006 & 0.007 \\
2016 General & 0.003 & 0.003 & 0.004 \\
2016 Primary & 0.005 & 0.005 & 0.006 \\
2014 General & 0.006 & 0.006 & 0.008 \\
2012 General & 0.004 & 0.004 & 0.005 \\
\hline RMSE: & & & \\
2018 General & 0.021 & 0.025 & 0.03 \\
2018 Primary & 0.094 & 0.099 & 0.092 \\
2016 General & 0.062 & 0.064 & 0.068 \\
2016 Primary & 0.196 & 0.194 & 0.199 \\
2014 General & 0.236 & 0.238 & 0.24 \\
\hline & 0.115 & 0.118 & 0.121 \\
\hline
\end{tabular}


Table SM8: Dropping Inattentive Respondents Reduces Bias in Voting-by-Mail Estimates

\begin{tabular}{lccc}
\hline & All & Pass IRI & Pass IMC \\
\hline Bias: & & & \\
2018 General & -0.055 & -0.048 & -0.044 \\
2018 Primary & -0.049 & -0.041 & -0.043 \\
2016 General & -0.07 & -0.062 & -0.061 \\
2016 Primary & -0.051 & -0.048 & -0.045 \\
2014 General & -0.056 & -0.052 & -0.047 \\
2012 General & -0.093 & -0.094 & -0.094 \\
\hline Std. Error: & & & \\
2018 General & 0.006 & 0.006 & 0.008 \\
2018 Primary & 0.007 & 0.008 & 0.01 \\
2016 General & 0.007 & 0.008 & 0.01 \\
2016 Primary & 0.008 & 0.009 & 0.011 \\
2014 General & 0.009 & 0.01 & 0.012 \\
2012 General & 0.009 & 0.01 & 0.012 \\
\hline RMSE: & & & \\
2018 General & 0.055 & 0.049 & 0.044 \\
2018 Primary & 0.05 & 0.041 & 0.044 \\
2016 General & 0.07 & 0.062 & 0.061 \\
2016 Primary & 0.052 & 0.049 & 0.047 \\
2014 General & 0.056 & 0.053 & 0.048 \\
2012 General & 0.093 & 0.094 & 0.095 \\
\hline & & &
\end{tabular}




\section{Supplementary Materials References}

Ansolabehere, S., and Hersh, E. 2017. Validation: What Big Data Reveal About Survey Misreporting and the Real Electorate. Political Analysis, 20(4), 437-459. Caughey, D., Berinsky, A. J., Chatfield, S., Hartman, E., Schickler, E., and Sekhon, J. S. 2020. Target Estimation and Adjustment Weighting for Survey Nonresponse and Sampling Bias New York: Cambridge University Press.

Gerber, A. S., and Green, D. P. 2000. The effects of canvassing, telephone calls, and direct mail on voter turnout: A field experiment. American Political Science Review, 94(3), 653-663.

Green, D. P. and Gerber, A. S. 2006. Can Registration-Based Sampling Improve the Accuracy of Midterm Election Forecasts? Public Opinion Quarterly, 70(2), $197-223$.

Hersh, E. D. 2015. Hacking the Electorate: How Campaigns Perceive Voters.. New York: Cambridge University Press. 